\title{
Perampanel: What is its Place in the Management of Partial Onset Epilepsy?
}

David R. M. Ledingham • Philip N. Patsalos

To view enhanced content go to www.neurologytherapy-open.com

Received: June 4, 2013 / Published online: August 30, 2013

(c) The Author(s) 2013. This article is published with open access at Springerlink.com

\section{ABSTRACT}

Introduction: Current pathways for treatment of partial onset epilepsy are diverse and include 14 new antiepileptic drugs (AEDs) licensed for use as either monotherapy or adjunctive therapy. However, the impact of these new AEDs on the treatment of partial epilepsy has so far been disappointing and there persists a need for additional drugs. Recently, perampanel, a first-in-class AED was licensed as an adjunct for the management of refractory partial onset seizures with or without secondary

D. R. M. Ledingham

Oxford University Hospitals NHS Trust, Oxford, UK

P. N. Patsalos $(\bowtie)$

Department of Clinical and Experimental Epilepsy,

UCL- Institute of Neurology, Queen Square,

London WC1N 3BG, UK

e-mail: P.Patsalos@ucl.ac.uk

P. N. Patsalos

Chalfont Centre for Epilepsy, Epilepsy Society,

Chalfont St Peter, Buckinghamshire, UK

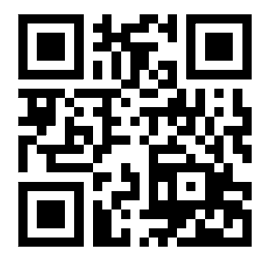

Enhanced content for this article is

available on the journal web site:

www.neurologytherapy-open.com generalization in patients 12 years and older. This review highlights the current management of partial epilepsy and analyses the published clinical and preclinical data of perampanel to consider its potential role in the treatment of partial epilepsy.

Methods: A literature review of Embase, Medline and PubMed was conducted in April 2013 using the search terms 'perampanel' and 'AMPA receptor antagonist/blocker'. Publications were included if they discussed perampanel in the context of preclinical or clinical epilepsy.

Results: Perampanel acts on the glutamate pathway. It is a novel highly selective noncompetitive alpha-amino-3-hydroxy-5-methyl4-isoxazolepropionic acid (AMPA) receptor antagonist. This is a previously untargeted post-synaptic glutamate receptor. It is responsible for mediating rapid trans-synaptic signal transduction and hence believed to play a major role in seizure propagation. The three pivotal placebo-controlled trials of adjunctive perampanel demonstrated that the effective dosing range is $4-12 \mathrm{mg} /$ day. The drug can be prescribed once daily, and its adverse effect profile is minimal with dizziness, fatigue, 
headache, and somnolence being the most commonly reported.

Conclusions: Perampanel is a welcome addition as it represents an alternative approach in the management of epilepsy with potential to have a significant impact on the prognosis of intractable epilepsy. However, it has only recently been licensed for clinical use in Europe, the USA, and Canada, and there are no data directly comparing it with other AEDs; hence, it remains far too early to ascertain its place in the treatment of patients with partial epilepsy.

Keywords: Adjunct therapy; AMPA receptor; First-in-class; Glutamate; Neurology; Noncompetitive AMPA receptor antagonist; Partial epilepsy; Perampanel; Refractory epilepsy

\section{INTRODUCTION}

Since 1989 there has been an exponential increase in the number of antiepileptic drugs (AEDs) used to treat patients with epilepsy, in general, and those with partial epilepsy in particular. In addition to the five firstgeneration AEDs (carbamazepine, phenobarbital, phenytoin, primidone, and valproate), there are ten second-generation AEDs (felbamate, gabapentin, lamotrigine, levetiracetam, oxcarbazepine, pregabalin, tiagabine, topiramate, vigabatrin, and zonisamide) and four third-generation AEDs (eslicarbazepine acetate, lacosamide, retigabine, and perampanel). In addition, there are two orphan AEDs (rufinamide and stiripentol), which are licensed for the treatment of specific 'difficult to treat' epilepsy syndromes.

Despite the hype, new AEDs have so far had minimal impact on the prognosis of intractable partial epilepsy in adults. Thus, whilst $63 \%$ of patients achieved seizure freedom in a cohort of patients with newly diagnosed epilepsy in 2000 [1], only 5\% more achieved seizure freedom 12 years later, despite the introduction of many new AEDs [2]. These new AEDs, however, are associated with improved adverse effect profiles and pharmacokinetic characteristics, particularly a reduced propensity to pharmacokinetic interactions, compared with early AEDs [3].

Recently a new AED, perampanel with a firstin-class mechanism of action, was approved in Europe, the USA, and Canada as adjunctive treatment of partial seizures with or without secondarily generalization in patients 12 years and older. The purposes of this review were to highlight the current management of partial epilepsy, to analyze the published clinical and preclinical data of perampanel, and to discuss its potential place in the treatment of partial epilepsy.

\section{METHODS}

Literature searches of Embase (from 1980 to April 2013), Medline (from 1950 to April 2013) and PubMed (from 1966 to April 2013) databases were conducted in April 2013. The search terms 'perampanel' and 'AMPA receptor antagonist/blocker' were used. Inclusion criteria included publications written in English, clinical, and preclinical studies/reviews that discussed the effects of perampanel or alphaamino-3-hydroxy-5-methyl-4-

isoxazolepropionic acid (AMPA) receptor antagonists in epilepsy, or in vitro/in vivo models of epilepsy. The above search terms identified 3,186 abstracts. Eighty-nine publications were selected on the basis of meeting the above inclusion criteria. Primary sources were preferred, but review articles were 
used in the absence of a primary reference. Data contained in summary of product characteristics (SPCs) were used whenever a published article was not available.

\section{DISCUSSION}

\section{Partial epilepsy}

Functionally seizures are divided into partial and generalized subtypes. Partial, or focal-onset seizures as they are also known, are thought to originate in a network of connections that facilitate seizure propagation constrained to one cerebral hemisphere [4]. This contrasts with the concept of generalized seizures, which are believed to rapidly engage a network involving both hemispheres [4]. Partial seizures may be simple seizures involving one area in a hemisphere, for example an isolated motor seizure, or can be more complex; there may be alternative networks of propagation through the hemisphere or networks that cross to involve both hemispheres giving rise to secondary generalized seizures.

Partial epilepsy is a diverse category. This is reflected by prevalence estimates, which vary markedly depending on the study population.
Twenty-five separate studies have looked at the relative prevalence of partial and generalized epilepsy [5]. In Europe, partial epilepsy accounts for between $18 \%$ and $63 \%[6,7]$ of cases, and in North America the figure varies from between $12 \%$ and $59 \%[8,9]$. The large variation in these figures is thought to reflect differences in partial epilepsy classification and study design between publications.

\section{Current Management of Partial Epilepsy}

A wide range of AEDs with differing mechanisms of actions are licensed for the treatment of partial epilepsy. The International League Against Epilepsy (ILAE) published an evidence review in 2013 highlighting 13 AEDs, which have been shown to have varying degrees of effectiveness as initial monotherapy [10] (Table 1).

The difficulty arises in establishing which AEDs should be used first, and in what order should therapeutic trials progress if initial monotherapy fails. In patients with refractory epilepsy, the issue then arises as to which AEDs are effective as an adjunct. One large health technology assessment carried out in 2006 concluded that there was little evidence to support the use of newer AEDs over older

Table 1 AEDs that have been shown to be effective in the first-line management of partial epilepsy [10]

\begin{tabular}{lll}
\hline Level of evidence & $\begin{array}{l}\text { Degree of effectiveness } \\
\text { in partial epilepsy }\end{array}$ & AED \\
\hline A & 'Established' & $\begin{array}{l}\text { Carbamazepine, levetiracetam, phenytoin, } \\
\text { and zonisamide }\end{array}$ \\
B & 'Probable' & Valproate \\
C & 'Possible' & $\begin{array}{l}\text { Gabapentin, lamotrigine, oxcarbazepine, phenobarbital, } \\
\text { topiramate, and vigabatrin }\end{array}$ \\
D & 'Potential' & Clonazepam and primidone \\
\hline
\end{tabular}

$A E D$ antiepileptic drug 
AEDs as monotherapy or adjuncts [11]. The assessment was unable to demonstrate consistently significant differences in AEDs with regard to efficacy or tolerability.

\section{First-Line AEDs for Partial Epilepsy}

In order to answer the question: 'what is the best first line AED in partial epilepsy?', the 'standard and new antiepileptic drugs study' (SANAD) was devised [12]. This was a large, unblinded, randomized controlled trial based in the UK. It recruited 1,721 patients and randomized them to receive carbamazepine, gabapentin, lamotrigine, oxcarbazepine, or topiramate. With regard to time to treatment failure, lamotrigine was significantly better than all alternative AEDs, with the exception of oxcarbazepine. In addition, carbamazepine was significantly better than alternative AEDs with regard to the proportion of patients experiencing remission at 12 months, although the advantage was non-significant when compared with lamotrigine. The authors concluded that although carbamazepine may be the more effective drug at maintaining seizure control, lamotrigine was clinically superior as it demonstrated better tolerability. The SANAD study is disputed by some researchers, but for many experts carbamazepine and lamotrigine are now considered first-line agents in the treatment of partial epilepsy.

If the first-line agent fails, then typically clinicians try a further two AEDs as monotherapy. AED choice is guided by multiple factors: (1) the implications of an AEDs' known adverse effect profile on patients, e.g., avoidance of strongly teratogenic agents in young women, (2) whether an AED has proven to be effective in specific target groups, e.g., the elderly or children, groups which have thus far undergone less investigation than middle-aged adults, (3) pharmacokinetic characteristics and drug-drug interaction profile, (4) a clinicians' experience of individual AEDs, and (5) the cost of AEDs.

\section{Refractory Partial Epilepsy}

In the past, the definition of refractory or drugresistant epilepsy varied widely in the academic literature. Only in 2010 did the ILAE propose a consensus statement defining drug-resistant epilepsy as 'failure of adequate trials of two tolerated and appropriately chosen and used AED schedules (whether as monotherapies or in combination) to achieve sustained seizure freedom' [13]. A long-term follow-up study of 1,098 patients, who initially started treatment in Scotland, identified that failure of seizure freedom despite multiple AEDs occurs in up to $30 \%$ of patients with epilepsy [2]. Of the total study population, $49.5 \%$ of patients became seizure-free on their first AED, a further 13.3\% on their second, $3.7 \%$ on their third, and successively smaller amounts when subsequent AEDs were trialed either as monotherapy or adjunct.

The typical pathway if monotherapy fails is to continue the AED that has proven most successful and add an adjunct. Cochrane reviews have demonstrated that clobazam [14], eslicarbazepine acetate [15], gabapentin [16], lamotrigine [17], levetiracetam [18], oxcarbazepine [19], tiagabine [20], topiramate [21], vigabatrin [22], and zonisamide [23] are all effective as adjunctive treatment of refractory partial seizures. However, there is little guidance from published literature as to what order these agents should be trialed, but many look to combinations with different mechanisms of action. What is known is that the actual placebo-corrected efficacy for AEDs as adjuncts is small $[24,25]$. A large meta-analysis 
incorporated the results of 54 studies, involving 11,106 patients [25], and demonstrated that, after correction for placebo, AEDs used as an adjunct in refractory epilepsy resulted in seizure freedom in only $6 \%$, and reduced seizure frequency by more than $50 \%$ in only $21 \%$ of patients.

In patients with epilepsy who have failed to respond to pharmacologic treatment surgery can be considered. Only a minority of patients are suitable. Surgery may range from a minimally invasive procedure, for example, insertion of a vagal nerve stimulator, to invasive intracranial surgery. The success rate varies depending upon the procedure: $66 \%$ of patients with temporal lobe resections will become seizure-free long term, whilst for subpial transections, the likelihood of seizure freedom is only $16 \%$ [26].

\section{Perampanel}

\section{Mechanism of Action}

The pathophysiology of seizure generation remains poorly understood. Essentially, a seizure represents an imbalance between neuronal excitation and inhibition. Synaptic concentrations of excitatory neurotransmitters such as glutamate rise, whilst inhibitory neurotransmitters such as gammaaminobutyric acid (GABA) fall [27]. Continuing with this simplification, current AEDs can be broadly divided into two groups: agents that act to inhibit excitatory mechanisms and those that act to promote inhibitory mechanisms [27] (in reality, many AEDs have been demonstrated to have multiple potential mechanisms of action).

Many AEDs including phenobarbital, tiagabine, topiramate, valproate, and vigabatrin have been demonstrated to act, at least in part, by promotion of the inhibitory
GABA pathway. In contrast, the excitatory pathway is thought to be inhibited at multiple different points by different AEDs. Two of the most commonly used AEDs in partial epilepsy act in this fashion, carbamazepine [28] and lamotrigine [29]. Their main antiepileptic activity arises from inhibition of sodium channels, which act to maintain neuronal membrane stability.

\section{Targeting Glutamate Pathways}

A more direct approach to blocking the glutamate pathway has been the subject of much research. Glutamate has a number of ionotropic and metabotropic receptors. The $\mathrm{N}$ methyl-D-aspartate (NMDA) and AMPA receptors are the most extensively explored within the context of epilepsy. NMDA receptors were the first target. However, initial results suggested limited antiepileptic activity, with epilepsy actually deteriorating in some patients [30]. In addition, the adverse effect profile was severe, with a significant proportion of patients developing frank psychosis.

Perampanel is the first licensed AED to act at AMPA receptors. It is a non-competitive antagonist as a result of binding to AMPA receptors at an allosteric site. This potentially accounts for part of its therapeutic action. Initial studies in rat seizure models compared NBQX and GYKI 52466, respectively, competitive and non-competitive antagonists at AMPA receptors [31]. Both were protective in several seizure models, but in models involving AMPA-induced seizures, the non-competitive inhibitor was effective, whereas the competitive inhibitor was not. Competitive antagonism to AMPA receptors means that at high glutamate concentrations, the antagonist can become displaced by glutamate, permitting channel opening, subsequent post-synaptic depolarization, and seizure propagation. High 
glutamate concentrations occur during seizures, meaning that competitive inhibitors are likely to fail at exactly the point when they are needed the most. Non-competitive antagonism means that inhibitory effects are less likely to be overwhelmed during seizures. Perampanel is a more soluble, non-competitive successor of these research agents [32].

In vitro studies have selectively blocked AMPA and NMDA receptors in neural tissues. NMDA receptor blockade has little effect on epileptiform discharge in fully kindled seizures, although it may shorten the discharge burst [33]. In contrast, selective AMPA receptor blockers have a marked inhibitory effect on epileptiform discharge, even in fully kindled seizure models. AMPA receptors permit sodium, potassium, and rarely calcium conductance, and are thought to be the means of rapid glutamatergic signal transduction [34]. NMDA receptors are blocked by magnesium at resting membrane potentials and require significant, sustained depolarization as provided by high frequency AMPA activity before they are activated [35]. They allow conductance of calcium as well as sodium and potassium. Calcium acts as a potent mediator of intracellular signal transduction. It initiates a chain of events responsible for long-term potentiation (LTP) [34, 35]. Disruption to LTP pathways is the proposed mechanism for the development of psychotic symptoms following human exposure to NMDA receptor antagonists. In contrast, AMPA receptor antagonists have less influence on LTP. They are proposed to mediate routine inter-neuronal synaptic communication, whilst NMDA receptors are responsible for longer term synaptic plasticity [34].

\section{Clinical Trial Data}

Perampanel has been studied within the context of two phase II clinical trials (Studies:
206 and 208) [36], three phase III clinical trials (Studies: 304, 305 and 306) [37-39] and two extension studies looking at long-term efficacy (Studies: 207 and 307) [40, 41].

\section{Efficacy}

Three phase III clinical trials assessed the efficacy of perampanel versus placebo across doses of $2-12 \mathrm{mg} / \mathrm{day}$. The studies were large, multicenter, multinational, double-blind randomized controlled trials that used intention to treat analysis. The majority of patients studied were young (mean ages varied from 33.4 to 36.7 years between the three studies; all patients were 12 years or older), Caucasian (61-86\%), had on average been diagnosed with epilepsy for $\sim 20$ years, and were considered refractory (average seizures 9-14/28 days, $70 \%$ secondarily generalized seizures). Patients had diagnoses of simple or complex partial seizures, with or without secondary generalization. More than $80 \%$ of patients had failed two or more AEDs in the previous 2 years and were on 1-3 concomitant AEDs (the most common of which were carbamazepine, lamotrigine, levetiracetam, and valproate). For trial inclusion, all participants had to have at least five partial seizures during the 6-week baseline period. The primary endpoints were the responder rate, defined as the percentage of patients exhibiting a $\geq 50 \%$ reduction in seizure activity, and the percentage change in seizure frequency. The main secondary endpoints were the median percentage change in seizure frequency over 28 days and seizure freedom.

All studies demonstrated a significant improvement in median change in seizure frequency with 4-12 mg/day perampanel. However, only two studies $(305,306)[38,39]$ showed a significant improvement in responder rate versus placebo (Table 2). A dose-dependent 
Table 2 The clinical effectiveness of perampanel versus placebo in published clinical trials

\begin{tabular}{lllll}
\hline $\begin{array}{l}\text { Perampanel } \\
\text { dose }(\mathbf{m g})\end{array}$ & Study & $\begin{array}{l}\text { Patients with } \mathbf{2 5 0 \%} \\
\text { reduction in seizure activity }\end{array}$ & $\begin{array}{l}\text { Median change in seizure } \\
\text { frequency over } \mathbf{2 8} \text { days }\end{array}$ & $\begin{array}{l}\text { TRAEs requiring } \\
\text { discontinuation of } \\
\text { perampanel }\end{array}$ \\
\hline 2 & 306 & $20.6 \%$ vs. $17.9 \%$ & $-13.6 \%$ vs. $-10.7 \%$ & $6.7 \%$ vs. $3.8 \%$ \\
4 & 306 & $28.5 \%$ vs. $17.9 \%^{*} p=0.0132$ & $-23.3 \%$ vs. $-10.7 \%^{*} p=0.0026$ & $2.9 \%$ vs. $3.8 \%$ \\
8 & 304 & $37.6 \%$ vs. $26.4 \% p=0.0760$ & $-26.3 \%$ vs. $-21 \%^{*} p=0.0261$ & $9 \%$ vs. $8 \%$ \\
& 305 & $33.3 \%$ vs. $14.7 \%^{*} p=0.002$ & $-30.5 \%$ vs. $-9.7 \%^{*} p<0.001$ & $9.3 \%$ vs. $4.4 \%$ \\
& 306 & $34.9 \%$ vs. $17.9 \%^{*} p=0.0003$ & $-30.8 \%$ vs. $-10.7 \%^{*} p<0.0001$ & $7.1 \%$ vs. $3.8 \%$ \\
12 & 304 & $36.1 \%$ vs. $26.4 \% p=0.0914$ & $-34.5 \%$ vs. $-21 \%^{*} p=0.0158$ & $26 \%$ vs. $8 \%$ \\
& 305 & $33.9 \%$ vs. $14.7 \%^{*} p<0.001$ & $-17.6 \%$ vs. $-9.7 \%^{*} p=0.011$ & $19 \%$ vs. $4.4 \%$ \\
\hline
\end{tabular}

Data taken from studies 304-306 [37-39]

TRAEs Treatment-related adverse events

* Statistically significant; $p$ value $<0.05$ compared with placebo

increment in responder rate and a median percentage change in seizure frequency were seen over $4-8 \mathrm{mg}$ /day doses. Doses of $2 \mathrm{mg}$ /day had no significant effect compared with placebo [39]. Compared with $8 \mathrm{mg} /$ day doses, $12 \mathrm{mg} /$ day had no increased effect in responder rates [37, 38] and showed an improvement in median percentage seizure frequency rates in only one of the two studies (305) that investigated perampanel at this higher dose [38]. Study 304, in which $12 \mathrm{mg} /$ day perampanel failed to make a difference in median percentage seizure frequency, also failed to show a change in responder rate at any dose compared with placebo.

Several explanations have been suggested for the lack of significant change in responder rate with perampanel in Study 304. French and colleagues proposed that the lack of impact may be due to: (1) the fact that the responder rate has a lower sensitivity when compared with median change in seizure frequency rendering significant differences harder to establish, or (2) the findings of Study 304 may have been skewed by its inclusion of Central and South American patients, who had a substantially higher placebo responder rate than the North American population, with some speculation as to whether the diagnosis of epilepsy was correct for some of these patients and if socio-economic factors played a part in the high placebo response [39]. Further analysis suggested that if one looked at just the North American group, then the responder rate at 8 and $12 \mathrm{mg} /$ day of perampanel differed significantly from placebo. This suggests problems with the implementation of the study in Central and South America. There do not appear to be any substantial differences in patient selection. In both studies 304 and 305, the proportion of patients with partial and complex partial seizures, the number of concomitant AEDs at baseline, baseline seizure frequency, and median time since diagnosis of epilepsy were similar. The only difference was that a higher proportion of patients in 305 were taking levetiracetam ( $\sim 38 \%$ in Study 305 versus $\sim 26 \%$ in Study 304 ). Thus, the potential for pharmacodynamic interactions between perampanel and alternative AEDs cannot be excluded.

An interim report from Study 307: a longterm, open-label extension of studies 304, 305 
and 306, demonstrated that the reduction in seizure frequency with perampanel was sustained long term (median duration of perampanel therapy of 51.4 weeks) [41]. However, the lack of placebo and no limitation on any additional AEDs added after the completion of the original phase III studies render this study difficult to interpret with regard to long-term efficacy.

\section{Treatment-Related Adverse Events Dis-} continuation from perampanel phase III clinical trials occurred in $6-19 \%$ of patients as a result of treatment-related adverse events (TRAEs). These events were dose dependent (Table 2). The majority of TRAEs were classified as mild-moderate with very few studies identifying severe events. The most common TRAEs in all trials, including extension studies, were dizziness, fatigue, headache, and somnolence [37-41]. The former three events in particular occurred in a dose-dependent fashion. The adverse events that most frequently resulted in cessation of perampanel or dose-reduction were ataxia, dizziness, convulsion, fatigue, headache, and vertigo [37-39]. None of the studies demonstrated a significant worsening of seizures compared with placebo, and only one case of sudden unexpected death in epilepsy occurred [42]. There were no deaths directly related to perampanel treatment. Weight increases of up to $\sim 2 \mathrm{~kg}$ were experienced with the highest doses of perampanel.

Psychiatric Adverse Events The most commonly reported behavioral TRAE was aggression. This increased in a dose-dependent fashion with $12 \mathrm{mg} /$ day doses resulting in aggression in up to $3.1 \%$ of patients, but resulting in cessation of therapy in only one case [42]. Although the data set is small, aggression appeared to be more common in adolescents $(n=22,18.2 \%)$ than in the overall population $(n=53,4.5 \%)$. Three adolescent patients (2.5\%) and 13 adult patients (1.1\%) withdrew due to aggression. Aggression was severe in 3 adolescents (2.5\%) compared with 8 patients in the overall population $(<1 \%)$. Reported adverse events were low and similar to placebo with regard to suicidality and other behavioral disorders. These tended to occur in patients with a strong personal history of mental health disorder and other potentially precipitating factors were normally identified, for example, recent cessation of risperidone in one patient.

Pharmacokinetic Profile Perampanel displays good oral bioavailability (100\%), is rapidly absorbed ( $T_{\max }, 0.25-2.0 \mathrm{~h}$ ) and demonstrates no sign of significant first-pass metabolism. It is $\sim 95 \%$ plasma protein bound and is widely distributed throughout tissues with a volume of distribution of $1.1 \mathrm{~L} / \mathrm{kg}$. Perampanel demonstrates linear pharmacokinetics in healthy individuals at doses of $2-12 \mathrm{mg} /$ day. It is extensively metabolized (98\%) primarily by CYP3A4 (although CYP3A5 may also contribute)-mediated oxidation and then undergoes sequential glucuronidation to produce various glucuronide conjugates. In the absence of CYP3A inducers, the half-life of perampanel in adult healthy volunteers is 51-129 h (mean $105 \mathrm{~h}$ ) after single dose and $66-90 \mathrm{~h}$ after multiple-dose administration $[43,44]$; this can be reduced to $\sim 25 \mathrm{~h}$ with strong enzyme inducers, e.g., carbamazepine. Seventy percentage of a perampanel dose is excreted in feces, the rest by the renal system [45]. Only $\sim 2 \%$ of an administered dose is excreted as unchanged perampanel in urine. The usual dosage of perampanel is $4-12 \mathrm{mg}$ / day. 
The pharmacokinetics of perampanel has important implications for its use. Its long half-life means that perampanel can be prescribed once daily, which will enhance patient compliance. Caution should be taken in the context of concomitant CYP3A4 inducers including AEDs such as carbamazepine, oxcarbazepine, phenytoin, and topiramate, and non-AEDs such as rifampicin (although no data on an effect of rifampicin are available) as they may significantly reduce circulating levels of perampanel (area under the curve [AUC] values are decreased by a mean 20-67\%), thereby reducing the responder rate. Similarly, the CYP3A inhibitor ketoconazole has been shown to elevate the mean plasma perampanel AUC values by $20 \%$. In contrast, perampanel had little impact on other AEDs, with the exception of oxcarbazepine where it increased, via an unknown mechanism, circulating plasma levels by $35 \%$. The clinical significance of this interaction is unknown because the pharmacologically active metabolite of oxcarbazepine, 10-hydroxycarbazepine, was not measured. In addition, perampanel is both hepatically and renally excreted; hence, caution should be taken in patients with hepatic and renal impairment.

\section{Perampanel's Potential in Management}

Pathways Perampanel has only been studied in humans within the context of refractory partial epilepsy in clinical trials of highly selected patients. Rat models of absence epilepsy have shown perampanel to be ineffective in this condition [46]. Published data regarding the effectiveness of perampanel in primary generalized epilepsies and as monotherapy for partial epilepsy are not available; thus, it is unclear how effective it could be in these settings. Post-hoc analyses of the original phase III clinical trials have shown that perampanel is significantly effective in the management of partial onset seizures with secondary generalization [47]. This is important as these patients are harder to manage than those with simple partial seizures. Perampanel demonstrated a clinically significant, dose-dependent rise in responder rate and median change in secondary generalized seizure activity at doses up to $8 \mathrm{mg} /$ day. This has been demonstrated for many older AEDs: carbamazepine [48], lacosamide [49], lamotrigine [50], levetiracetam [51], phenytoin [48], topiramate [52], and valproate [48], but little published data are available with respect to the ability of alternative newer AEDs in this context.

Perampanel's application in the management of pediatric epilepsy has yet to be established. The original trials contained small numbers of adolescents aged $>12$ years. Aside from a suggested increase in the risk of behavioral side effects in adolescent compared with adult patients [42], little information with regard to relative efficacy and rates of other TRAEs is known.

\section{CONCLUSIONS}

During the last two decades, 14 AEDs have been specifically licensed for the treatment of intractable partial epilepsy but with little impact on the prognosis of such patients overall, although for some individual patients seizure freedom has been achieved. An ongoing observational study by Brodie and colleagues has looked at the number of patients who go on to become seizure-free following their initial diagnosis of epilepsy. The group has published a series of follow-up analyses over the course of a decade. In the first study published in 2000, $63 \%$ of patients ultimately became seizure-free [1], in the latest study published in 2012 this 
figure was $68 \%$ [2]. That is a disappointing increment of only $5 \%$ over 12 years, in spite of the introduction of these new AEDs.

Intractable partial epilepsy is an epilepsy subtype crying out for ground-breaking new drugs. Perampanel's novel mechanism of action as a non-competitive AMPA receptor antagonist represents a new and previously unexplored target. Furthermore, its non-competitive antagonism means that at high glutamate concentrations, perampanel's inhibitory effects are less likely to fail and consequently it may be particularly useful in refractory patients. In addition, clinical trials have demonstrated that perampanel is effective in refractory partial epilepsy and that it has a relatively mild adverse effect profile.

There is, however, as yet no trial data comparing perampanel with alternative AEDs. The difficulty for clinicians remains how to pick one adjunct over another. As perampanel has only recently been licensed for clinical use, and since there are no data directly comparing perampanel with other AEDs, it remains far too early to ascertain the place of perampanel in the treatment of patients with epilepsy.

\section{ACKNOWLEDGMENTS}

The work of Philip Patsalos was supported by the National Institute for Health Research University College London Hospitals Biomedical Research Centre. Prior to peer review, Eisai were offered the opportunity to review this paper for scientific accuracy. No writing assistance, other editorial involvement, or financial support was provided by the manufacturer for this study or publication of this article. This article does not necessarily reflect the opinions, policies, or recommendations of Eisai or any of its employees.
Conflict of interest. David Ledingham declares that he has no conflict of interest. Philip Patsalos has received speaker and consultancy fees from Eisai, the manufacturer of perampanel.

Open Access. This article is distributed under the terms of the Creative Commons Attribution Noncommercial License which permits any noncommercial use, distribution, and reproduction in any medium, provided the original author(s) and the source are credited.

\section{REFERENCES}

1. Kwan P, Brodie MJ. Early identification of refractory epilepsy. N Engl J Med. 2000;342:314-9.

2. Brodie MJ, Barry SJ, Bamagous GA, Norrie JD, Kwan P. Patterns of treatment response in newly diagnosed epilepsy. Neurology. 2012;78:1548-54.

3. Johannessen LC, Patsalos PN. Drug interactions involving the new second and third generation antiepileptic drugs. Expert Rev Neurother. 2010;10:119-40.

4. Berg AT, Berkovic SF, Brodie MJ, et al. Revised terminology and concepts for the organization of seizures and epilepsies: report of the ILAE Commission on Classification and Terminology, 2005-2009. Epilepsia. 2010;51:676-85.

5. Banerjee PN, Filippi D, Hauser WA. The descriptive epidemiology of epilepsy-a review. Epilepsy Res. 2009;85:31-45.

6. Guiliani G, Terziani S, Senigaglia AR, Luccioni G, Foschi N, Maffei C. Epilepsy in an Italian community as assessed by a survey for prescriptions of antiepileptic drugs: epidemiology and patterns of care. Acta Neurol Scand. 1992;85:23-31.

7. Luengo A, Para J, Colas J, et al. Prevalence of epilepsy in northeast Madrid. J Neurol. 2001;248:762-7.

8. Haerer AF, Anderson DW, Schoenberg BS. Prevalence and clinical features of epilepsy in a biracial United States population. Epilepsia. 1986;27:66-75. 
9. Hauser WA, Annegers JF, Kurland LT. Prevalence of epilepsy in Rochester, Minnesota: 1940-1980. Epilepsia. 1991;32:429-45.

10. Glauser T, Ben-Menachem E, Bourgeois B, et al. Updated ILAE evidence review of antiepileptic drug efficacy and effectiveness as initial monotherapy for epileptic seizures and syndromes. Epilepsia. 2013;54:551-63.

11. Wilby J, Kainth A, Hawkins $\mathrm{N}$, et al. Clinical effectiveness, tolerability and cost-effectiveness of newer drugs for epilepsy in adults: a systematic review and economic evaluation. Health Technol Assess. 2005;9:1-157, iii-iv.

12. Marson AG, Al-Kharusi AM, Alwaidh M, et al. The SANAD study of effectiveness of carbamazepine, gabapentin, lamotrigine, oxcarbazepine or topiramate for treatment of partial epilepsy: an unblinded randomised controlled trial. Lancet. 2007;24;369:1000-15.

13. Kwan P, Arzimanoglou A, Berg AT, et al. Definition of drug resistant epilepsy: consensus proposal by the ad hoc Task Force of the ILAE Commission on Therapeutic Strategies. Epilepsia. 2010;51:1069-77.

14. Michael B, Marson AG. Clobazam as an add-on in the management of refractory epilepsy. Cochrane Database Syst Rev. 2008;2:CD004154.

15. Chang $X$, Yuan $H$, Wang $Y$, Xu H, Zheng R. Eslicarbazepine acetate add-on for drug-resistant partial epilepsy. Cochrane Database Syst Rev. 2011;12:CD008907.

16. Marson AG, Kadir ZA, Hutton JL, Chadwick DW. Gabapentin add-on for drug-resistant partial epilepsy. Cochrane Database Syst Rev. 2000;3:CD001415.

17. Ramaratnam S, Marson AG, Baker GA. Lamotrigine add-on for drug-resistant partial epilepsy. Cochrane Database Syst Rev. 2001;3:CD001909.

18. Mbizvo GK, Dixon P, Hutton JL, Marson AG. Levetiracetam add-on for drug-resistant focal epilepsy: an updated Cochrane Review. Cochrane Database Syst Rev. 2012;9:CD001901.

19. Castillo S, Schmidt DB, White S. Oxcarbazepine add-on for drug-resistant partial epilepsy. Cochrane Database Syst Rev. 2000;3:CD002028.

20. Pulman J, Marson AG, Hutton JL. Tiagabine add-on for drug-resistant partial epilepsy. Cochrane Database Syst Rev. 2012;5:CD001908.

21. Jette $\mathrm{N}$, Hemming $\mathrm{K}$, Hutton JL, Marson AG. Topiramate add-on for drug-resistant partial epilepsy. Cochrane Database Syst Rev. 2008;3: CD001417.

22. Hemming K, Maguire MJ, Hutton JL, Marson AG. Vigabatrin for refractory partial epilepsy. Cochrane Database Syst Rev. 2013;1:CD007302.

23. Chadwick DW, Marson AG. Zonisamide add-on for drug-resistant partial epilepsy. Cochrane Database Syst Rev. 2005;4:CD001416.

24. Schmidt D. Efficacy of new antiepileptic drugs. Epilepsy Curr. 2011;11:9-11.

25. Beyenburg S, Stavem K, Schmidt D. Placebocorrected efficacy of modern antiepileptic drugs for refractory epilepsy: systematic review and metaanalysis. Epilepsia. 2010;51:7-26.

26. Tellez-Zenteno JF, Dhar R, Wiebe S. Long-term seizure outcomes following epilepsy surgery: a systematic review and meta-analysis. Brain. 2005;128:1188-98.

27. Besag FM, Patsalos PN. New developments in the treatment of partial-onset epilepsy. Neuropsychiatr Dis Treat. 2012;8:455-64.

28. Willow $M$, Catteral WA. Inhibition of binding of $\left[{ }^{3} \mathrm{H}\right]$ batrachotoxinin A 20- $\alpha$-benzoate to sodium channels by the anticonvulsant drugs diphenylhydantoin and carbamazepine. Mol Pharmacol. 1982;22:627-35.

29. Lang DG, Wang CM, Cooper BR. Lamotrigine, phenytoin and carbamazepine interactions on the sodium current present in N4TG1 mouse neuroblastoma cells. J Pharmacol Exp Ther. 1993;266:829-35.

30. Sveinbjornsdottir S, Sander JW, Upton D, et al. The excitatory amino acid antagonist S-CPP-ene (SDZ EAA-494) in patients with epilepsy. Epilepsy Res. 1993;16:165-74.

31. Yamaguchi S, Donevan SD, Rogawaski MA. Anticonvulsant activity of AMPA/kainate antagonists: comparison of GYKI 52466 and NBOX in maximal electroshock and chemoconvulsant seizure models. Epilepsy Res. 1993;15:179-84.

32. Hanada $\mathrm{T}$, Hashizume $\mathrm{Y}$, Tokuhara $\mathrm{N}$, et al. Perampanel: a novel, orally active, noncompetitive AMPA-receptor antagonist that reduces seizure activity in rodent models of epilepsy. Epilepsia. 2011;52:1331-40.

33. Traus RD, Miles R, Jeffreys JG. Synaptic and intrinsic conductances shape picrotoxin-induced synchronized after discharges in the guinea-pig hippocampal slice. J Physiol. 1993;461:525-47. 
34. Rogawski MA. Revisiting AMPA receptors as an antiepileptic drug target. Epilepsy Curr. 2011;11:56-63.

35. Collingridge GL, Isaac JT, Wang YT. Receptor trafficking and synaptic plasticity. Nat Rev Neurosci. 2004;5:952-62.

36. Krauss GL, Bar M, Biton V, et al. Tolerability and safety of perampanel: two randomised doseescalation studies. Acta Neurol Scand. 2012;125:8-15.

37. French JA, Krauss GL, Biton V, et al. Adjunctive perampanel for refractory partial-onset seizures: randomized phase III study 304. Neurology. 2012;79:589-96.

38. French JA, Krauss GL, Steinhoff BJ, et al. Evaluation of adjunctive perampanel in patients with refractory partial-onset seizures: results of randomized global phase III study 305. Epilepsia. 2013;54:117-25.

39. Krauss GL, Serratosa JM, Villanueva V, et al. Randomized phase III study 306: adjunctive perampanel for refractory partial-onset seizures. Neurology. 2012;78:1408-15.

40. Rektor I, Krauss GL, Bar M, et al. Perampanel Study 207: long-term open-label evaluation in patients with epilepsy. Acta Neurol Scand. 2012;126:263-9.

41. Krauss GL, Perucca E, Ben-Menachem E, et al. Perampanel, a selective, noncompetitive $\alpha$-amino3-hydroxy-5-methyl-4-isoxazolepropionic acid receptor antagonist, as adjunctive therapy for refractory partial-onset seizures: interim results from phase III, open-label extension study 307. Epilepsia. 2013;54:126-34.

42. Serratosa JM, Villanueva V, Kerling F, Kasper BS. Safety and tolerability of perampanel: a review of clinical trial data. Acta Neurol Scand. 2013;127: 30-5.

43. Perampanel (Fycompa). Summary of Product Characteristics. 2012. Eisai Europe Ltd. Available at: http://www.ema.europa.eu/docs/en_GB/docu ment_library/EPAR_-_Product_Information/human/ 002434/WC500130815.pdf. Accessed July 31, 2013.
44. Plosker GL. Perampanel: as adjunctive therapy in patients with partial-onset seizures. CNS Drugs. 2012;26:1085-96.

45. Committee for Medicinal Products for Human Use. Fycompa (perampanel) assessment report. European Medicines Agency 424476, 2012. Available at: http://www.ema.europa.eu/docs/en GB/document_library/EPAR_-_Public_assessment_ report/human/002434/WC500130839.pdf. Accessed July 31, 2013.

46. Kamiński RM, Van Rijn CM, Turski WA, Czuczwar SJ, Van Luijtelaar G. AMPA and GABA(B) receptor antagonists and their interaction in rats with a genetic form of absence epilepsy. Eur J Pharmacol. 2001;430:251-9.

47. Ko D, Ramsay RE. Perampanel: expanding therapeutic options for patients with medically refractory secondary generalised convulsive seizures. Acta Neurol Scand. 2013;127:36-43.

48. Ramsay RE, Detoledo J. Tonic-clonic seizures: a systematic review of antiepilepsy drug efficacy and safety. Clin Ther. 1997;19:433-46.

49. Chung S, Sperling MR, Biton V, et al. Lacosamide as adjunctive therapy for partial-onset seizures a randomised controlled trial. Epilepsia. 2010;51: 958-67.

50. Schapel GJ, Beran RG, Vajda FJ, et al. Double-blind, placebo controlled, crossover study of lamotrigine in treatment resistant partial seizures. J Neurol Neurosurg Psychiatry. 1993;56:448-53.

51. Shorvon SD, Lowenthal A, Janz D, Bielen E, Loiseau P. Multicentre double-blind, randomized placebocontrolled trial of levetiracetam as add-on therapy in patients with refractory partial seizures. European Levetiracetam Study Group. Epilepsia. 2000;41:1179-86.

52. Ben-Menachem E, Henrikson O, Dam M, et al. Double-blind, placebo-controlled trial of topiramate as add-on therapy in patients with refractory partial seizures. Epilepsia. 1996;37: 539-43. 